\title{
Fractional abundances of molecules in dense interstellar clouds: A compendium of recent model results ${ }^{\star}$
}

\author{
H.-H. Lee ${ }^{1}$, R.P.A. Bettens ${ }^{1}$ and E. Herbst ${ }^{1,2}$ \\ 1 Department of Physics, The Ohio State University, Columbus, OH 43210, U.S.A. \\ 2 Department of Astronomy, The Ohio State University, Columbus, OH 43210, U.S.A.
}

Received January 16; accepted February 21, 1996

\begin{abstract}
In this paper we present calculated fractional abundances in dense interstellar clouds for selected atomic and molecular species using three different homogeneous, pseudo-time-dependent models discussed by Bettens, Lee, \& Herbst (1995): the new standard model, the new neutral-neutral model, and model 4 . We have run each model with 3 different hydrogen densities $-10^{3}, 10^{4}$, and $10^{5} \mathrm{~cm}^{-3}$ - and two temperatures - $10 \mathrm{~K}$ and $50 \mathrm{~K}$. "Low metal" elemental abundances have been used for all three models; the new standard model has also been run with "high metal" abundances.
\end{abstract}

Key words: ISM: clouds — ISM: abundances — ISM: molecules — molecular processes

\section{Introduction}

The history of gas-phase chemical models of dense interstellar clouds is a long and varied one. The first such model was constructed by Herbst \& Klemperer (1973), who used a homogeneous, steady-state approach and included 100 chemical reactions involving 35 species up to $4-5$ atoms in size. The limited agreement with observational abundances obtained by Herbst \& Klemperer (1973) indicated that ion-molecule reactions are an important mechanism for the synthesis of gas-phase molecules in dense clouds. This conclusion was also reached by Watson $(1973,1974)$. An improved steady-state gas-phase model was subsequently obtained by Mitchell et al. (1978), while the synthesis of larger molecules was considered by Mitchell \& Huntress (1979), Suzuki (1983), Herbst et al. (1984), and Millar \& Freeman (1984) among others.

In 1980, Prasad \& Huntress (1980a, b) introduced the so-called "pseudo-time-dependent" approach to the chemical modeling of dense interstellar clouds. They solved the simultaneous differential kinetic equations for the gasphase chemistry as a function of time with a given set of initial abundances and fixed, homogeneous physical conditions. With this approach, steady-state abundances are reached in about $10^{7}$ years. In the absence of an effective desorption mechanism, however, this time is significantly longer than the time needed for species other than

Send offprint requests to: E. Herbst

* Tables 2-13 are only available in electronic form at the CDS via anonymous ftp 130.79.128.5
$\mathrm{H}_{2}$ and $\mathrm{He}$ to be depleted from the gas phase onto the mantles of dust grains (Boland \& De Jong 1982). The time is also larger than the free-fall time against gravitational collapse (Langer \& Glassgold 1976). Langer \& co-workers (Graedel et al. 1982; Langer et al. 1984) utilized and modified the approach of Prasad \& Huntress (1980a, b) to study the dependence of the calculated abundances on assumed gas phase elemental abundances and to investigate the isotopic fractionation of heavy isotopes. They also pointed out that the use of theoretical abundances at times well before steady state is reached can be justified. Leung et al. (1984), Herbst \& Leung (1986a, b, 1989, 1990), and Bettens et al. (1995) developed this point of view and greatly expanded the pseudo-time-dependent model to contain nearly 4000 gas-phase reactions involving over 400 species through 13 atoms in size. These authors emphasized that the densities of organic molecules peak at a so-called "early time" of $10^{4.5}-10^{5.5} \mathrm{yr}$, at which time the abundances are in good agreement with the observed abundances for many species in dense cloud condensations such as TMC-1 and the extended ridge in Orion, especially if the "low metal" elemental abundances recommended by Graedel et al. (1982) are utilized. The abundances of large organic molecules decline to quite low values as steadystate conditions are reached. The analysis contains the assumption that the initial abundances are atomic except for molecular hydrogen. Extensions of the time-dependent gas-phase models to include grain chemistry have been attempted (e.g. Hasegawa \& Herbst 1993) but the results are 
quite uncertain and the dust chemistry has been handled inadequately (Charnley \& Tielens, in preparation).

The formation of large molecules in pseudo-timedependent models via gas phase pathways is very sensitive to the unknown contribution of neutral-neutral reactions (Millar \& Nejad 1985; Millar et al. 1987; Bettens \& Brown 1992; Herbst et al. 1994). Recent laboratory work (Sims \& Smith 1995) has shown that certain classes of neutralneutral reactions can occur rapidly at very low temperature, but the extent of this phenomenon is still not determined. In general, the inclusion of rapid neutral-neutral reactions tends to depress the calculated early-time abundances of complex molecules, sometimes catastrophically (Herbst et al. 1994). Bettens et al. (1995) have shown that the rates of reactions between $\mathrm{O}$ or $\mathrm{N}$ atoms and carbon clusters $\mathrm{C}_{n}$ are critical among unstudied classes of neutralneutral reactions. They concluded that if $\mathrm{O}+\mathrm{C}_{n}$ and $\mathrm{N}$ $+\mathrm{C}_{n}$ are slow, then complex molecules can be produced in high abundance despite the existence of other rapid neutral-neutral reactions.

Although homogeneous pseudo-time-dependent models are still quite popular and are available for observers to use (Farquhar \& Millar 1993), dynamic models in which the physical parameters change as the chemistry progresses have also been pursued (Gerola \& Glassgold 1978; Tarafdar et al. 1985; Nejad et al. 1990), occasionally with dust chemistry. This type of global approach has given way to models in which the influence of local star formation on chemistry through gravitational collapse and thermal warming is emphasized (Brown et al. 1988; Willacy et al. 1994). Models of so-called 'Hot Molecular Cores' rely on grain chemistry to produce saturated organic molecules such as methyl formate not readily produceable in the gas (Brown et al. 1988; Caselli et al. 1993). The existence of young and massive stars inside dense clouds leads to surrounding HII regions, in which atomic ions dominate, but somewhat farther away from these stars, molecules exist despite relatively high photon fluxes in so-called photondominated regions, models of which have been around for a decade (Tielens \& Hollenbach 1985; Sternberg \& Dalgarno 1995).

The growing evidence that dense interstellar clouds are inhomogeneous over a range of distance scales (van Dishoeck et al. 1993) has led to new generations of chemical models. One class of models shows that the non-linear kinetic equations lead to two solutions - the low ionization phase heretofore discussed, and a high ionization phase - which coexist for certain regions of parameter space (Pineau des Forêts et al. 1992; Le Bourlot et al. 1993, 1995). Thus, depending on history, there may exist different chemical states of the gas at different points in dense clouds. Another class of models considers mixing of material between dense and diffuse clumps of gas. For example, Chièze et al. (1991) have utilized such dynamical mixing to show that an enhancement of both neutral carbon and complex molecule abundances can occur compared with homogeneous models at steady state.

Even in sources where cloud interiors are not dominated by stellar formation, and where clumping of material is moderate, the gas will increase in density and visual extinction with increasing depth into the cloud. Slab models of dense interstellar clouds have been contructed to take the more rarefied outer and intermediate layers of material into account along with the interior material. Xie et al. (1995) used a 22-slab model with 1-D geometry and variable density to study the effect of turbulent diffusion in the time-dependent gas-phase chemistry of small molecules. Bergin et al. (1995) used a 30-zone 1-D model with constant density to study the role of grain adsorption and desorption on gas-phase abundances. Most recently, Lee et al. (1996) constructed a 43-slab model with variable density and constant temperature. The radiative transfer in outer layers, which includes attenuation due to grains as well as to the self- and cross-shielding of $\mathrm{H}_{2}$ and $\mathrm{CO}$, is treated in detail in their model. They also consider the gas-phase chemistry of large molecules and the contributions of external and intermediate slabs to atomic and molecular abundances.

Despite the general trend of models towards increasingly spatial complexity, there still exists a role for homogeneous pseudo-time-dependent models. These simple models are often the first consulted by observers when considering observations of new molecules or after having detected them. We have not presented a complete list of calculated abundances using such models since 1989 (Herbst \& Leung 1989), during which time the reaction network used has undergone significant changes. The changes were discussed by Bettens et al. (1995), but these authors only presented limited results for a small sample of organic molecules. Besides being of importance to observers, the results of homogeneous models should be viewed as a baseline, from which one can determine how other factors change calculated abundances. Accordingly, in this paper, we present a large number of results from our latest homogeneous pseudo-time-dependent models.

In the following sections, the chemical models used for the calculations are explained, and the results are briefly discussed. The calculated abundances are presented in 12 tables; Table 1 contains a list of initial non-zero abundances relative to total hydrogen used in most of the calculations, while Tables 2-13 contains lists of the fractional abundances (with respect to $\mathrm{H}_{2}$ ) of selected species at differing temperatures and gas densities. All tables but the first are presented in electronic format, and can be accessed by anonymous ftp at 130.79.128.5 (CDS).

\section{Chemical models}

The calculated abundances presented here derive from three models with rapid ion-dipolar neutral rates discussed in Bettens et al. (1995): the new standard model, 
Table 1. Initial fractional abundances with respect to total hydrogen for low metals case

\begin{tabular}{lc}
\hline Element & Abundance \\
\hline $\mathrm{He}$ & $1.4000(-1)$ \\
$\mathrm{N}$ & $2.1400(-5)$ \\
$\mathrm{O}$ & $1.7600(-4)$ \\
$\mathrm{C}^{+}$ & $7.3000(-5)$ \\
$\mathrm{S}^{+}$ & $8.0000(-8)$ \\
$\mathrm{Si}^{+}$ & $8.0000(-9)$ \\
$\mathrm{Fe}^{+}$ & $3.0000(-9)$ \\
$\mathrm{Na}^{+}$ & $2.0000(-9)$ \\
$\mathrm{Mg}^{+}$ & $7.0000(-9)$ \\
$\mathrm{e}$ & $7.3107(-5)$ \\
$\mathrm{P}^{+}$ & $3.0000(-9)$ \\
$\mathrm{Cl}^{+}$ & $4.0000(-9)$ \\
\hline
\end{tabular}

the new neutral-neutral model, and model 4 . The new standard model is an extension of the gas-phase dense cloud model of Herbst \& Leung (1989), which has been updated by including 133 new species, two new elements$\mathrm{Cl}$ and $\mathrm{P}$ - and many new reactions, including a comprehensive sulphur chemistry (Millar \& Herbst 1990) and a complete (if uncertain) list of cosmic ray-induced photodestruction reaction rates. Some of the revisions were sent to us by C.M. Leung, and some derive from the UMIST data base (Farquhar \& Millar 1993). The direct cosmic ray ionization rate $\zeta$ is $1.310^{-17} \mathrm{~s}^{-1}$. The new standard model contains 3785 gas-phase reactions and 409 species through 13 atoms in size. Comparison of the early-time results from this model using "low metal" abundances with observed abundances for a wide variety of molecules in TMC-1 shows excellent agreement (Herbst 1996).

The new neutral-neutral model has been developed from the new standard model by adding certain classes of neutral-neutral reactions, estimated to be rapid by extension of the small set of relevant laboratory measurements (Sims \& Smith 1995). This model represents a more comprehensive attempt to incorporate rapid neutral-neutral reactions than the model along the same lines described in Herbst et al. (1994). Use of this model leads to very low calculated abundances for complex molecules at early time, a point discussed in detail by both Herbst et al. (1994) and Bettens et al. (1995). The model 4 described in the work of Bettens et al. (1995) has been developed from the new neutral-neutral model by deleting 2 classes of neutral-neutral reactions critical to the destruction of complex species $-\mathrm{O}+\mathrm{C}_{n}$ and $\mathrm{N}+\mathrm{C}_{n}$. Recent theoretical work on the prototype reaction between $\mathrm{O}$ and $\mathrm{C}_{3}$ shows that the reaction is indeed very slow at low temperature (Woon \& Herbst, ApJ, in press).

As shown in Bettens et al. (1995), model 4 produces large abundances of complex molecules but is not in as good agreement with observations in TMC-1 as is the new standard model, which is still the model recommended for observers until more information is available concerning the rates of neutral-neutral reactions at low temperatures. Readers who desire any of the three reaction networks should contact one of the authors.

The non-hydrogenic non-zero relative abundances adopted here for most model calculations are listed in Table 1. All hydrogen is assumed to be initially in its molecular phase. These abundances add depleted values for $\mathrm{Cl}$ and P to the "low metal" abundances (Graedel et al. 1982) in which the elements $\mathrm{S}, \mathrm{Si}, \mathrm{Na}, \mathrm{Mg}$, and Fe are depleted by two orders of magnitude. There is more oxygen than carbon, so the chemistry used here is "oxygen-rich". With the exception of hydrogen, helium, oxygen, and nitrogen, all elements are intially present in singly charged atomic form. Calculations have been run at two different temperatures $-10 \mathrm{~K}$ and $50 \mathrm{~K}$ - representing dark clouds (e.g. TMC-1) and extended regions of giant clouds, respectively, and for each temperature we have used three different gas densities $n=n_{\mathrm{H}}+2 n_{\mathrm{H}_{2}}: 10^{3}, 10^{4}$, and $10^{5}$ $\mathrm{cm}^{-3}$. Since "high metal" abundances may pertain to certain regions, we have also run a series of calculations with the new standard model in which the abundances for $\mathrm{S}$, $\mathrm{Si}, \mathrm{Na}, \mathrm{Mg}$, and $\mathrm{Fe}$ are two orders of magnitude greater, although $\mathrm{Cl}$ and $\mathrm{P}$ have been kept at their low values. The visual extinction has been set sufficiently high that photoprocesses caused by external photons do not occur, although cosmic ray-induced processes are fully considered. The time-dependent results have been obtained by solving a system of ordinary differential equations with the Gear method. With the elemental abundances chosen, there is only one solution, representing the so-called low ionization phase. This does not rule out the possibility of other steady-state solutions.

\section{Results}

Our results are listed in twelve tables (Table 2 - Table 13). Each table contains atomic and molecular abundances with respect to $\mathrm{H}_{2}$ at both $10 \mathrm{~K}$ and $50 \mathrm{~K}$. The ordering scheme of the species is as follows: i) neutrals are listed before ions, ii) molecules in each class are listed in increasing size order, and iii) molecules with a given number of atoms are listed generally alphahetically but with standard chemical formulae (e.g., OCS rather than COS). The abundances are listed at times of $10^{5} \mathrm{yr}, 10^{5.5} \mathrm{yr}$, $10^{6} \mathrm{yr}$, and steady state for the two lower densities and at times of $10^{4.5} \mathrm{yr}, 10^{5} \mathrm{yr}, 10^{5.5} \mathrm{yr}$, and steady state for the highest density. Tables $2-4$ contain results from the new standard model at the three different densities, Tables 57 do the same for the new neutral-neutral model, while Tables $8-10$ repeat the process for model 4 . Tables $11-13$ contain our results for the new standard model with "high metal" abundances. The criterion for choosing a species to be listed is that its fractional abundance must be over 
$10^{-12}$ in any of the 18 model runs with "low metal" abundances. This criterion leads to the listing of 280 atomic and molecular species in addition to the density of negativelycharged grains.

A study of the model results shows that the new standard model with low metals comes the closest to the earlier but smaller low metal models of Herbst \& Leung (1989, 1990) although the inclusion of cosmic ray-induced photoreactions can change results by factors of a few. Larger changes occur for species affected directly by measured or calculated changes in reaction rates which have occurred during the last 6 years, as occurs, for example, in the sulfur chemistry (Millar \& Herbst 1990). Large changes in the sulfur chemistry are quite pronounced when high metal abundances are used in the new standard model.

In addition to very low predictions for the early-time abundances of complex molecules, the new neutral-neutral results show a large decrease in the radical $\mathrm{CN}$, which has been measured to be surprisingly reactive at low temperature. Neither of these changes is in agreement with observations in standard sources. The model 4 results show sizeable amounts of complex molecules but the diminution in abundance with increasing molecular size in a given family (e.g., $\mathrm{C}_{n} \mathrm{H}, \mathrm{HC}_{2 n} \mathrm{CN}$ ) is smaller than both that observed in TMC1 and that calculated via the new standard model. The synthesis of large molecules in model 4 is heavily dependent on neutral-neutral reactions. Extrapolated to larger molecules than contained in the results reported here, model 4 leads to predictions of large abundances for some truly complex hydrocarbons (Bettens \& Herbst, submitted).

The dependence on density of the assorted results in the studied range is complex. In general, radicals and ions decrease in fractional abundance with increasing density whereas stable molecules can increase. To complicate matters, peak early-time abundances tend to shift to shorter times as density increases. This shift should be considered if it is desired to interpolate the tabulated results.

Acknowledgements. E.H. acknowledges the support of the National Science Foundation for his research program in astrochemistry. He also thanks the Ohio Supercomputer Center for time on their Cray YMP-8 machine, without which these calculations would not have been possible.

\section{References}

Bergin E.A., Langer W.D., Goldsmith P.F., 1995, ApJ 441, 222 Bettens R.P.A., Brown R.D., 1992, MNRAS 258, 347

Bettens R.P.A., Lee H.-H., Herbst E., 1995, ApJ 443, 664

Boland W., De Jong T., 1982, ApJ 261, 110

Brown P.D., Charnley S.B., Millar T.J., 1988, MNRAS 231, 409
Caselli P., Hasegawa T.I., Herbst E., 1993, ApJ 408, 548

Chièze J.-P., Pineau des Forêts G., Herbst E., 1991, ApJ 373, 110

Farquhar P.R.A., Millar T.J., 1993, CCP7 Newslett. 18, 6

Gerola H., Glassgold A.E., 1978, ApJS 37, 1

Graedel T.E., Langer W.D., Frerking M.A., 1982, ApJS 48, 321

Hasegawa T.I., Herbst E., 1993, MNRAS 263, 589

Herbst E., 1996, Ion-Molecule Chemistry in Interstellar Clouds: Successes and Problems. In: Adams N.G., Babcock L.M. (eds.) Advances in Gas Phase Ion Chemistry, Vol. III. JAI, Greenwich, CT. (in press)

Herbst E., Adams N.G., Smith D., 1984, ApJ 285, 618

Herbst E., Klemperer W., 1973, ApJ 185, 505

Herbst E., Leung C.M., 1986a, MNRAS 222, 689

Herbst E., Leung C.M., 1986b, ApJ 310, 378

Herbst E., Leung C.M., 1989, ApJS 69, 271

Herbst E., Leung C.M., 1990, A\&A 233, 177

Herbst E., Lee H.-H., Howe D.A., Millar T.J., 1994, MNRAS 268,335

Langer W.D, Glassgold A.E., 1976, A\&A 48, 395

Langer W.D., Graedel T.E., Frerking M.A., Armentrout P. B., 1984, ApJ 277, 581

Le Bourlot J., Pineau des Forêts G., Roueff E., 1993, ApJ 416, L87

Le Bourlot J., Pineau des Forêts G., Roueff E., 1995, A\&A 297, 251

Lee H.-H., Herbst E., Pineau des Forêts G., Roueff E., Le Bourlot J., 1996, A\&A (to be published)

Leung C.M., Herbst E., Huebner W.F., 1984, ApJS 56, 231

Millar T.J., Freeman A., 1984, MNRAS 207, 405

Millar T.J., Herbst, E., 1990, A\&A 231, 466

Millar T.J., Leung C.M., Herbst E., 1987, A\&A 183, 109

Millar T.J., Nejad L.A.M., 1985, MNRAS 217, 507

Mitchell G.F., Ginsburg J.L., Kuntz P.J., 1978, ApJS 38, 39

Mitchell G.F., Huntress W.T.Jr., 1979, Nat 278, 722

Nejad L.A.M., Williams D.A., Charnley S.B., 1990, MNRAS 246, 183

Pineau des Forêts G., Roueff E., Flower D.R., 1992, MNRAS 258,45

Prasad S.S., Huntress W.T.Jr., 1980a, ApJS 43, 1

Prasad S.S., Huntress W.T.Jr., 1980b, ApJ 239, 151

Sims I.R., Smith I.W.M., 1995, Ann. Rev. Phys. Chem. 46, 109

Sternberg A., Dalgarno A., 1995, ApJS 99, 565

Suzuki H., 1983, ApJ 272, 579

Tarafdar S.P., Prasad S.S., Huntress W.T.Jr., Villere K.R., Black D.C., 1985, ApJ 289, 220

Tielens A.G.G.M., Hollenbach D., 1985, ApJ 291, 722

van Dishoeck E., Blake G.A., Draine B.T., Lunine J.I., 1993, in: Levy E.H., Lunine J.I. (ed.). U. of Arizona Press, Protostars and Planets III, p. 163

Watson W.D., 1973, ApJ 183, L17

Watson W.D., 1974, ApJ 188, 35

Willacy K., Rawlings J.M.C., Williams D.A., 1994, MNRAS 269,921

Xie T., Allen M., Langer W.D., 1995, ApJ 440, 674 